\title{
Applying the Long-Term Memory Algorithm to Forecast Loss of Thermoregulation Capacity in Honeybee Colonies
}

\author{
Antonio Rafael Braga ${ }^{1,3}$, Lia Sucupira Furtado ${ }^{1}$, Antonio Diego M. Bezerra ${ }^{2}$, \\ Breno M. Freitas ${ }^{2}$, Joseph A. Cazier ${ }^{3}$, Danielo G. Gomes ${ }^{1}$ \\ ${ }^{1}$ Grupo de Redes de Computadores, Engenharia de Software e Sistemas (GREat) \\ Departamento de Engenharia de Teleinformática (DETI) \\ Universidade Federal do Ceará (UFC), Fortaleza - CE, CEP 60455-970, Brasil. \\ ${ }^{2}$ Setor de Abelhas, Departamento de Zootecnia, Centro de Ciências Agrárias \\ Universidade Federal do Ceará (UFC), Fortaleza - CE, CEP 60.356-000, Brasil. \\ ${ }^{3}$ Center for Analytics Research and Education (CARE) \\ Computer Information Systems \& Supply Chain Management (CIS \& SCM) \\ Appalachian State University (ASU), Boone, NC, 28608-2037, United States \\ \{liafurtado, antoniobraga\}@great.ufc.br \{antonniodiego\}ealu.ufc.br \\ \{freitas, danielo\}@ufc.br cazierja@appstate.edu
}

\begin{abstract}
Bees are the main pollinators of most wild and cultivated plant species, thus being essential for the maintenance of plant ecosystems and for food production. But they are threatened due to a series of drivers such as pesticides, habitat loss and climate change. Here, we propose a method to identify the loss of thermoregulation capacity in honeybee colonies. We applied the Long Short-Term Memory (LSTM) algorithm, which is based on Recurrent Neural Networks (RNN), to six real datasets of the Arnia remote hive monitoring system. From brood temperatures gathered along the European fall season in 2017, the LSTM was able to detect when a honeybee colony is about to lose its thermoregulation capacity. Our results showed an error of only $0.5 \%$ in prediction for well-thermoregulated beehives.
\end{abstract}

\section{Introduction}

Pollinators are among the most important biotic agents and the pollination service they provide ensures ecosystems stability, maintenance of the biodiversity of wild plants species and agricultural yield [Potts et al. 2016, Bezerra et al. 2019]. However, pollinators have declined considerably in agricultural landscapes, and pollinator decline affects significantly the reproduction of plant species, crop production, food security and human well-being [Potts et al. 2010, Garibaldi et al. 2013].

Bees are the most important group of pollinators [Klein et al. 2007, Brown et al. 2016] and the Western honeybee (Apis mellifera) is the species most commonly used for pollination purpose around the world [Gallai et al. 2009, Potts et al. 2016]. But honeybee colonies have dwindled in many parts of the globe and average rates of $30 \%$ colony deaths overwinter have become common in recent years. Many drivers can be blamed for these figures such as diseases, weak workers due to parasites, low population for overwintering, workers with low fat body reserves, low honey stores [IPBES 2016]. 
Whatever the causes, they all converge to the same point; harming the thermoregulation capacity of the colonies and killing bees of cold decimating the entire colony or producing weak colonies next spring. As a consequence, the pollination service carried out by honeybees as well as their honey production is threatened every year. It is clear that protecting the population of honey bees (A. mellifera) worldwide, as well as enabling them to maximize their productivity, is an important concern [Edwards-Murphy et al. 2016].

To mitigate this concern, we apply the Long Short-Term Memory (LSTM) algorithm to forecast the thermoregulation capacity (i.e. homeostasis) loss in honeybee colonies. Here, we used datasets collected from the Arnia ${ }^{1}$ remote hive monitoring system as illustrated in Figure 1.

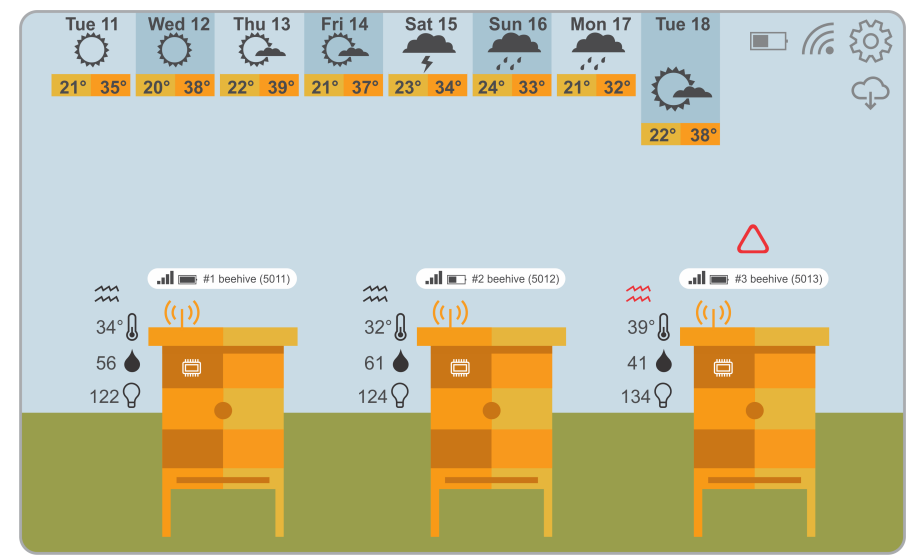

Figure 1. Dashboard overview of a general remote beehive monitoring system.

\section{Related works}

The thermoregulation capacity loss in beehives may be associated with different health problems in the colony. For example, the Bee Counted initiative ${ }^{2}$ supported by BroodMinder ${ }^{3}$ identified 5 phenomena directly related to thermoregulation: (i) brood onset for a new bee package, (ii) brood onset for an overwintering hive, (iii) second brood box coming online, (iv) small colony unable to fully thermoregulate and (v) queen loss. Nevertheless, each phenomenon was identified through the analysis of a specialist in beekeeping (i.e., non-automatically). However, unwanted phenomena, such as queen loss, can be extremely dangerous to colony health and survival and, ideally, an automatic detection should be much more useful to the beekeeper.

In tropical climate conditions, it is possible to observe bee abandonment scenarios due to the internal hive temperature. For example, it is possible to recognize thermal patterns to detect bees' pre-abandonment scenarios [Kridi et al. 2016]. In temperate climate conditions, the hotter the bees perform the foraging, which impacts the development of the pupae and the division of labor of the bees [Winston 1991]. Thus, the presence of all stages of brood can be affected by ambient (external) temperature.

The seasons of the year are also very important to the colony behavior [Maciel et al. 2018]. Over thousands of years, honey-bees have developed mechanisms

\footnotetext{
${ }^{1}$ http://www.arnia.co.uk

${ }^{2}$ https://www.beecounted.org/

${ }^{3}$ https://broodminder.com/
} 
to control the internal temperature of the nest, both in the hottest period of summer or when the outside temperature is very low $\left(\right.$ e.g., $\left.0^{\circ} \mathrm{C}\right)$, as in the winter in temperate regions [Winston 1991]. Among other mechanisms developed, we may mention (i) ventilation or waving of wings if the intention is to conduct hot air out of the colony and (ii) heating by pooling and metabolic heat generation if the purpose is to keep the colony warm.

Thus, the loss of internal temperature control is an important indicator related to the colony health, and may indicate if it is facing a problem. In this paper, we used machine learning techniques to predict homeostasis loss. The use of machine learning has become widespread in the analysis of beekeeping data [de Carvalho et al. 2018].

\section{Material and methods}

\subsection{Dataset}

The datasets used are from six different beehives and they were obtained from an apiary located in the city of Newcastle upon Tyne, England, distant 277 miles $(446 \mathrm{~km})$ from north London. They were collected from September to November in 2017, along the fall. The data has 7 features; (i) brood temperature (internal temperature), (ii) internal humidity, (iii) hive activity, (iv) mean fanning, (v) mean flight noise, (vi) weight, and (vii) external temperature. The internal temperature of the beehive and the external temperature has been measured in Celsius. The Table 1 presents a summary of sampling performed for each beehive, including the number of samples in each dataset. In the column "therm." (i.e, thermoregulation) w.r. means well regulated and n.r. means not regulated.

Table 1. Summary of beehive analyzed with good thermoregulation

\begin{tabular}{c|c|c|c|c|c|c}
\hline beehives & latitude & longitude & \#samples & period & sampling & therm. \\
\hline 9803 & -1.628 & 54.971 & 603 & Sep 3th-Nov 6th & $2 \mathrm{hs}$ & w.r. \\
9837 & -1.516 & 54.994 & 1456 & Sep 3th-Nov 6th & $1 \mathrm{~h}$ & n.r. \\
9841 & -1.617 & 54.979 & 638 & Sep 5th-Nov 6th & 2hs & n.r. \\
9848 & -1.599 & 55.016 & 502 & Sep 5th-Nov 2th & 2hs & n.r. \\
54440 & -1.628 & 54.971 & 606 & Sep 3th-Nov 6th & 2hs & n.r. \\
54460 & -1.616 & 54.970 & 1024 & Aug 5th-Nov 6th & 2hs & w.r. \\
\hline
\end{tabular}

The data was collected using the software Arnia. Arnia system uses three sensors inside the hive to collect the attributes that describe the internal conditions of the hive. A temperature sensor, a humidity sensor, a digital scale, and a sound sensor (microphone). The attributes can be grouped by objective, hive homeostasis (brood temperature/humidity), bee activity (flight/foraging and fanning acoustics) and productivity (hive weight). The colonies under study are of the species Apis mellifera.

\subsection{Preprocessing}

Initially, the Exploratory Data Analysis (EDA) using statistical methods was performed, in order to familiarize with the data and to maximize the retrieval of information hidden in its structure. After that, it was calculated basic statistics (mean, the standard deviation, and quartiles), and the skewness. Lastly, we performed the detection and removal of outliers and the scaling of the data set by the min-max method. 


\subsection{Detection and removal of anomalies}

Taken this analysis, the plotted boxplot of the dataset showed that there were some outliers in the internal temperature. To detect which were the outliers it was used the interquartile range, which is the difference between quartiles 1 and 3 of the data set. The numbers that were in the quartiles outside of this range were transformed into NULL. Because the dataset wasn't to big, it was chosen to impute the places that used to be anomalies. The imputation method chosen was of the mean, which replaces the missing value with the mean of the feature.

\subsection{Data Resizing (Min-Max scaling)}

Since LSTMs are sensitive to the scale of the input data, the data was resized to be used in the algorithm. Also, this is generally used to improve the numerical stability of a model. The min-max technique takes the values of the attributes in their original intervals and resizes them to a range of $[0,1]$. This can be useful in some cases where all parameters need to have the same positive scale. The samples set was normalized by the Equation 1 below:

$$
x_{n e w}=\frac{x-x_{\min }}{x_{\max }-x_{\min }} .
$$

\subsection{Long Short-Term Memory (LSTM)}

A Recurrent Neural Network (RNN) is a type of artificial neural network designed to recognize patterns in sequences of data. For this, they take into account data that was perceived previously in time. They do this by that feedback loop connected to their past decisions, ingesting their own outputs moment after moment as input. It is often said that recurrent networks have memory.

Long Short-Term Memory (LSTM) is a specific RNN architecture that was designed to model temporal sequences and their long-range dependencies more accurately than conventional RNNs [Sak et al. 2014]. As any Neural Network it has neurons and their connections with each other, every connection has a weight that is calculated through Back Propagation. Back Propagation moves backward from the final error through the outputs, weights, and inputs of each hidden layer, assigning those weights responsibility for a portion of the error and recalculating different weights until it finds the composition that minimizes the error.

In a neural network, the leftmost layer of the network is called the input layer, and the rightmost layer the output layer. The middle layer of nodes is called the hidden layer, because its values are not observed in the training set. In the LSTM architecture, the hidden layer has LSTM cells. As shown in Figure 2, each cell has 3 gates: an input gate, which controls if the memory cell is updated, a forget gate that controls if the cell is reset to zero, and an output gate that controls rather the information of the cell is made visible. This way information can be stored in, written to, or read from a cell, much like data in a computer's memory.

\subsection{Experiment Setup}

Our approach started by choosing a dataset among the six options of beehive's dataset. Afterwards it was performed a min-max scaling in the data so that distincts ranges of data 


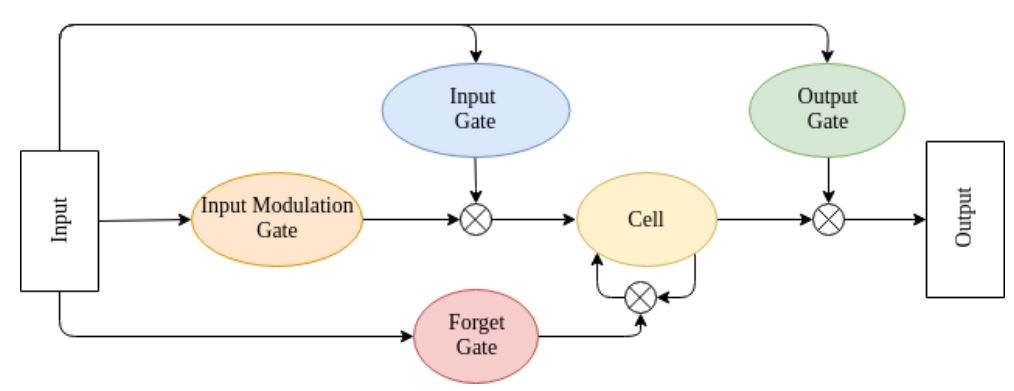

Figure 2. A LSTM memory cell

did not affect the results. Then the data was separated in train and test sets, with $67 \%$ being the data to be trained and $33 \%$ the data to be tested.

The LSTM model was build in a sequential manner by adding the layers of the neural network to create an ideal model. The model that best suited this problem was composed of a LSTM architecture with 4 hidden layers, a Dropout layer that is used to prevent overfitting and a Dense Layer that is a fully connected layer, meaning all the neurons in this layer are connected to those in the next layer.

To fit the model above in our dataset it was chosen the number of times the dataset would be passed forward and backward through the neural net refitting the weights everytime. Since LSTM is a iterative process, updating the weights with a single pass or one epoch is not enough. This would cause underfitting, where the model would not predict the values. However, a big epoch number could cause an overfitting because the weights would be so fixed for that particular dataset that it would not be able to predict alternatives ones. In sume, an epoch 100 was find ideal.

Moreover, to fit the model the back propagation parameter was set. This parameter is called look back and it represents the number of previous timestamps that are used by the model to predict the next timestamp. Initially, it was tested at 2, however this number didn't capture the complete pattern of the time series so the look back was setted in 4 timestamps.

With this, we fitted the model in the training dataset so that it could learn the patterns of the data.

Later on, the model predicted the results of the train and the test data, this way we obtained the predictions of our algorithm. To evaluate our model it was chosen a few metrics that will be detailed at section 3.7. This process was repeated for all six datasets, that way we could evaluate how the model created performed in datasets with different patterns, of a well-thermoregulated beehive or not.

\subsection{Evaluation Metrics}

In order to to summarize and evaluate the quality of the proposed solution, 4 metrics were used: (i) Mean Absolute Error (MAE), (ii) Mean Absolute Percentage Error (MAPE), (iii) Root Mean Squared Error (RMSE), and $\mathrm{R}^{2}$ (coefficient of determination), defined as:

(i) MAE is the mean for all recorded absolute errors.

(ii) MAPE is the mean absolute percentage error. 
(iii) RMSE measures the average of the squares of the errors, that is, the average squared difference between the estimated values and what is estimated.

(iv) $\mathrm{R}^{2}$ provides a measure of how well observed outcomes are replicated by the model, based on the proportion of total variation of outcomes explained by the model.

The metrics MAE, RMSE, and MAPE will show low values for a good model and $\mathrm{R}^{2}$ will give high values to indicate a more precisely predictive model.

\section{Results}

Initially, the algorithm was executed in 4 different beehives which were not thermoregulating well.

The first beehive analyzed was the 9837 which was chosen because of the downward trend of the internal temperature. Measures of the internal temperature starts around $36^{\circ} \mathrm{C}$ and dropped to $20^{\circ} \mathrm{C}$. The algorithm was executed to predict the internal temperature and to check if the colony has lost control of the temperature. The next beehive analyzed was the 9841, Figure 4, which pattern is a bit different of 9837 because despite its, overall descending temperature, the colony tried unsuccessfully to restore and maintain its temperature to the normal range.

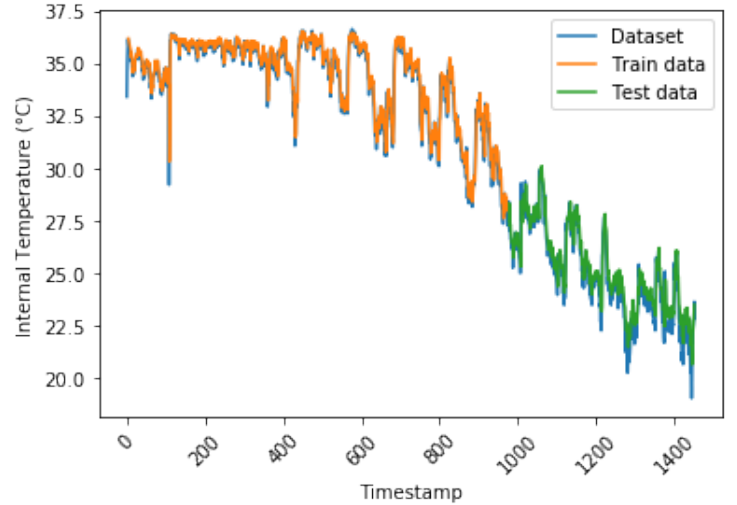

Figure 3. LSTM fitting (hive \#9837)

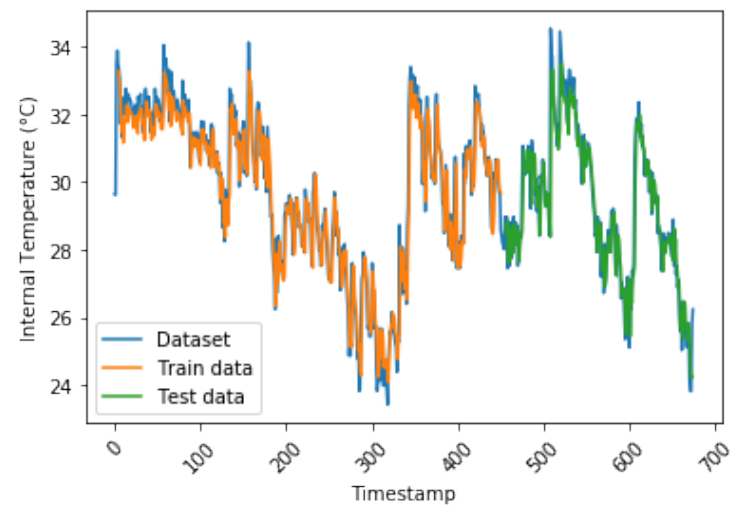

Figure 4. LSTM fitting (hive \#9841)

In the graphs present in Figures 3 and 4, the blue line is the original data, the orange part is the training dataset, and the green is the test. Upon looking at these graphs we can see that the model follows the descending pattern and the wide temperature amplitude variation pretty well. As shown in Table 2, we can see the results of the algorithm in these two beehives in the form of some metrics. The RMSE shows small values, as expected, and analyzing the MAPE we can see that the algorithm only showed around $2 \%$ of error when trying to fit the model in these datasets. So, the model predicts the outcome with a satisfactory performance.

The algorithm follows the pattern and predicts satisfactory temperature and the descendant nature of the beehives internal temperature. The model predicts the outcome with a satisfactory performance. Figures 5 and 6 show the regression obtained for beehives 9848 and 54440. It is possible to see that the internal temperature starts around $35^{\circ} \mathrm{C}$ and drops to around $12^{\circ} \mathrm{C}$ in beehive 9848 and $15^{\circ} \mathrm{C}$ in beehive 54440 . In these beehives, it is possible to observe a very accentuated variation on internal temperatures 
and the colonies can not perform the thermoregulation. In Section 5, the likely causes for this loss of control will be discussed.

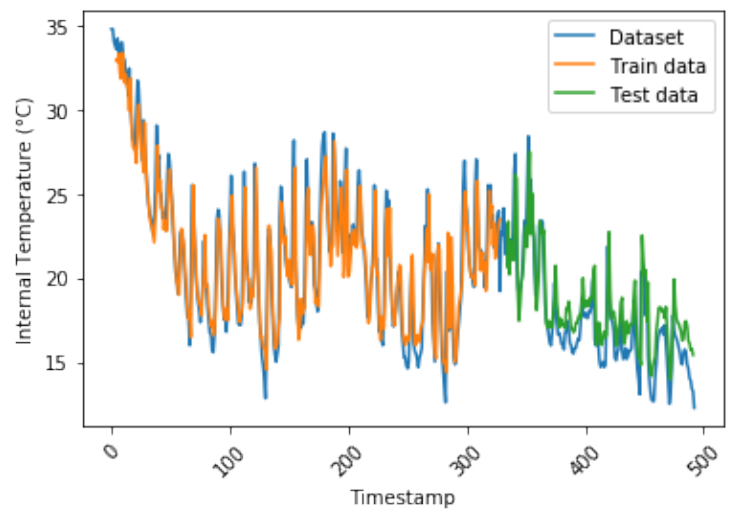

Figure 5. LSTM fitting (hive \#9848)

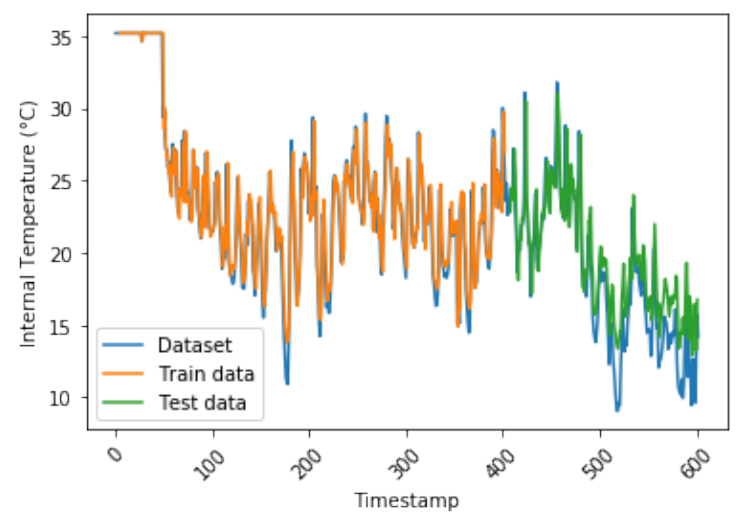

Figure 6. LSTM fitting (hive \#54440)

Looking at the values calculated for metrics in Table 2 for these two beehives, it is noticeable that the RMSE is bigger compared to the beehives 9837 and 9841 , but it is still a small value. This can be explained because of the intense variation in a large amplitude of temperature. Also, the $\mathrm{R}^{2}$ is smaller, but with these values we can say that approximately more than three fifths of the observed variation can be explained by the model's inputs.

Table 2. Evaluation Metrics for LSTM Algorithm for not thermoregulated beehives

\begin{tabular}{c|c|c|c|c|c}
\hline Beehive & RMSE Train & RMSE Test & MAE & MAPE & $\mathrm{R}^{2}$ \\
\hline 9837 & 0.49 & 0.73 & 0.5651 & 2.3424 & 0.8876 \\
9841 & 0.77 & 0.83 & 0.5658 & 1.9388 & 0.8543 \\
9848 & 1.79 & 1.72 & 1.4282 & 8.3675 & 0.6978 \\
54440 & 1.74 & 2.46 & 2.0794 & 13.614 & 0.7427 \\
\hline
\end{tabular}

Then, we chose two beehives, 9803 and 54460 which were capable of maintaining the temperature in a range of $33^{\circ} \mathrm{C}$ to $36^{\circ} \mathrm{C}$, which is considered a healthy range. The same model with the parameters configured and described above was set to execute the algorithm for these beehives.

In the beehive 9803 , Figure 7 , we had an internal temperature always in a range of a range of $32.4^{\circ} \mathrm{C}$ to $36^{\circ} \mathrm{C}$ meaning that the colony was capable of performing good thermoregulation and achieving homeostasis. Even thought this temperature has a descendent pattern because of the slow progression rate of fall, it does not represent any risk to the colony. Moreover, one can see from Table 3, that the metrics of RMSE, MAE and MAPE are very small values, for example, with a MAPE of only $0.5 \%$. So, the algorithm predicted well the temperatures in the range which was previously cited.

In regard to the beehive 54460 (Figure 8), its colony faced a great fall in the internal temperature but managed to rise temperature back and stabilize around $32^{\circ} \mathrm{C}$ to $36^{\circ} \mathrm{C}$. The algorithm was executed in this dataset and the results showed good evaluation metrics with only 0.64 RMSE (Table 3). This indicates that the model detects a rise in the temperature and predicts satisfactorily when the temperature rises and stabilizes. The values calculated for metrics can be seen in the Table 3 . 


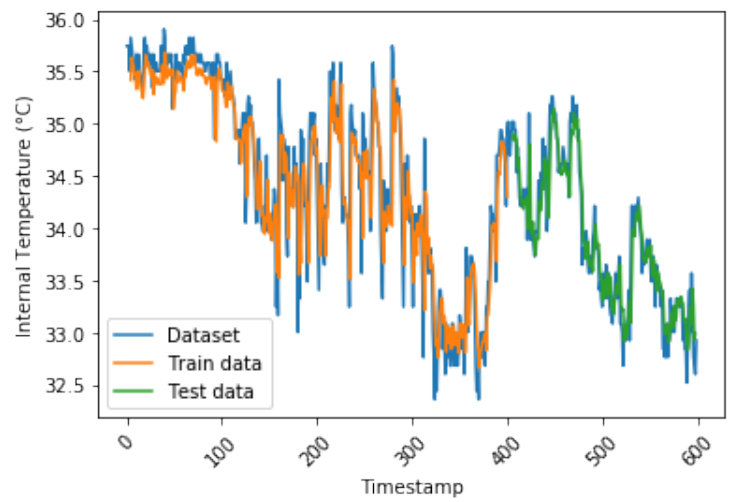

Figure 7. LSTM fitting (hive \#9803)

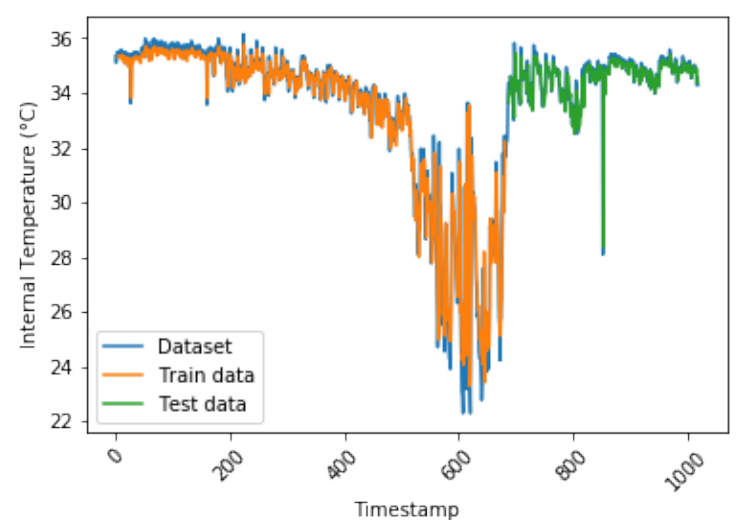

Figure 8. LSTM fitting (hive \#54460)

Table 3. Evaluation Metrics for LSTM Algorithm for thermoregulated beehives

\begin{tabular}{c|c|c|c|c|c}
\hline Beehive & RMSE Train & RMSE Test & MAE & MAPE & $\mathrm{R}^{2}$ \\
\hline 9803 & 0.38 & 0.25 & 0.1854 & 0.5478 & 0.8677 \\
54460 & 0.93 & 0.64 & 0.3102 & 0.9098 & 0.1276 \\
\hline
\end{tabular}

\section{Discussion}

We have shown that the algorithm could predict the amplitude and values of temperature and assess the state of beehives whether it is capable or not of thermoregulating.

The high amplitude of temperature and a declining pattern indicate a sign of thermoregulation losses. According to [Heldmaier 1987] the colony capacity to survive cold depends on maintenance of a steady state temperature, about $35^{\circ} \mathrm{C}$, in the colony's central brood area. In the datasets used in this work the season in which the data was collected through sensorring was the fall. During fall the temperature gradually drops, especially at night, in preparation for winter. So it was expected that the internal temperature would also falls following the external one. However, given the fact that in some beehives the temperature fell more than $10^{\circ} \mathrm{C}$, this could already be a sign of trouble, that could lead to several problems in the colony health.

If the internal temperature is too low the consequence is the brood dying off and the nectar not dehydrating fast enough to make honey. In extreme conditions of cold weather the bees take a protective behavior and start a phonomena called the diapause. It is a gradual and progressive interruption in development or ontogeny of the bees in order to survive these unfavorable environmental conditions[Winston 1991].

Another case of loss in the thermoregulation capability can be a beehive with high temperatures. The overheating happens when the beehive temperature is up to $36^{\circ} \mathrm{C}$, and it can lead to the brood death, wax melting and the honey getting dehydrated too quickly [Winston 1991].

Also, in our work we showed beehives where the inhabitant bee colony thermoregulated well and our algorithm can also predict this pattern. An appropriate control in temperature is crucial for the survival of the honeybees and the reproduction in this species. The effects of our results are of importance to the beekeeper's everyday work because 
he/she will be able to prepare or even prevent this problem before it happens. Besides that, the beekeeper will be able to adapt the handling of the colonies to prepare them, improving their micro-climate according to the results given from the prediction.

\section{Conclusion}

Our results showed it is possible to foresee when honeybee colonies lose their ability to thermoregulate. The algorithm used was efficient in predicting loss of homeostasis in the nest some time in advance. The algorithm was configured to use 4 predicted timestamps, and in the case of the hives here monitored, the proposed solution is capable of predicting the trend of thermoregulatory capacity loss of a colony up to 8 hours before the homeostasis is lost in the nest.

Therefore, a beekeeper will have a good prediction of the moment temperature begins to fall inside the nest demonstrating the bees are no longer capable of thermoregulate the nest. It is worth mentioning that the algorithm used performs predictions in time series. Thus, with the trained algorithm, it can be used in other hives to predict eminent states of loss of thermoregulatory control.

As future perspectives, we intend to inform others features to LSTM, like internal humidity, hive activity, mean fanning, mean flight noise, weight, external temperature, and external humidity to take the patterns of inability to thermoregulate and define the possible reasons for it happens in a specific environment. We also plan to create an interface allowing to notify in advance beekeepers when any of the colonies begin to loose its thermoregulating abilities, thus, the beekeeper can intervene and also determine causes that lead to loss of thermoregulation and apply the best practice to help the colony.

\section{Acknowledgements}

This study was financed in part by the Coordenação de Aperfeiçoamento de Pessoal de Nível Superior-Brasil (CAPES) - Finance Code 001. Dataset for this study comes from the Arnia remote hive monitoring system. The authors thank the valuable technical support of Lucas Noleto. Danielo G. Gomes and Breno Freitas thanks the financial support of the CNPq (Conselho Nacional de Desenvolvimento Científico e Tecnológico-Brasil, processes \#302934/2010-3, \#311878/2016-4, \#432585/2016-8, \#129426/2018-0).

\section{References}

Bezerra, A. D. M., Pacheco Filho, A. J., Bomfim, I. G., Smagghe, G., and Freitas, B. M. (2019). Agricultural area losses and pollinator mismatch due to climate changes endanger passion fruit production in the Neotropics. Agricultural Systems, 169(C):49-57.

Brown, M. J. F., Dicks, L. V., Paxton, R. J., Baldock, K. C. R., Barron, A. B., Chauzat, M. P., Freitas, B. M., Goulson, D., Jepsen, S. J., Kremen, C., Li, J., Neumann, P., Pattemore, D. E., Potts, S. G., Schweiger, O., Seymour, C. L., and Stout, J. (2016). A horizon scan of future threats and opportunities for pollinators and pollination. PeerJ, 4:2249.

de Carvalho, H. V. F., Carvalho, E. C., Arruda, H., Imperatriz-Fonseca, V., de Souza, P., and Pessin, G. (2018). Detecção de anomalias em comportamento de abelhas utilizando redes neurais recorrentes. In $9^{\circ}$ Workshop de Computação Aplicada a Gestão 
do Meio Ambiente e Recursos Naturais (WCAMA_CSBC 2018), volume 9, Porto Alegre, RS, Brasil. SBC.

Edwards-Murphy, F., Magno, M., M. Whelan, P., O’Halloran, J., and Popovici, E. (2016). B+wsn: Smart beehive with preliminary decision tree analysis for agriculture and honey bee health monitoring. Computers and Electronics in Agriculture, 124:211219.

Gallai, N., Sallesc, J.-M., Setteled, J., and Vaissièrea, B. E. (2009). Economic valuation of the vulnerability of world agriculture confronted with pollinator decline. Ecological Economics, 68:810-821.

Garibaldi, L. A. et al. (2013). Wild pollinators enhance fruit set of crops regardless of honey bee abundance. Science (New York, N.Y.), 339(6127):1608-1611.

Heldmaier, G. (1987). Temperature control in honey bee colonies. BioScience, 37:395399.

IPBES (2016). Summary for policymakers of the assessment report of the intergovernmental science-policy platform on biodiversity and ecosystem services on pollinators, pollination and food production. Bonn, Germany. S.G. Potts, V. L. Imperatriz-Fonseca, H. T. Ngo, J. C. Biesmeijer, T. D. Breeze, L. V. Dicks, L. A. Garibaldi, R. Hill, J. Settele, A. J. Vanbergen, M. A. Aizen, S. A. Cunningham, C. Eardley, B. M. Freitas, N. Gallai, P. G. Kevan, A. Kovács-Hostyánszki, P. K. Kwapong, J. Li, X. Li, D. J. Martins, G. Nates-Parra, J. S. Pettis, R. Rader, and B. F. Viana (eds.). Secretariat of the Intergovernmental Science-Policy Platform on Biodiversity and Ecosystem Services, Bonn, Germany.

Klein, A. M., Vaissiére, B. E., Cane, J. H., Steffan-Dewenter, I., Cunningham, S. A., Kremen, C., and Tscharntke, T. (2007). Importance of pollinators in changing landscapes for world crops. Proceedings. Biological sciences, 274 1608:303-313.

Kridi, D., Carvalho, C., and Gomes, D. (2016). Application of wireless sensor networks for beehive monitoring and in-hive thermal patterns detection. Computers and Electronics in Agriculture, 127:221-235.

Maciel, F. A. O., Braga, A. R., de Lima e Silva, A., da Silva, T. L. C., Freitas, B. M., and Gomes, D. G. (2018). Reconhecimento de padrões de colônias de abelhas apis mellifera segundo mudanças das estações do ano. In $9^{\circ}$ Workshop de Computação Aplicada a Gestão do Meio Ambiente e Recursos Naturais (WCAMA_CSBC 2018), volume 9, Porto Alegre, RS, Brasil. SBC.

Potts, S., Biesmeijer, J., Kremen, C., Neumann, P., Schweiger, O., and Kunin, W. (2010). Global pollinator declines: Trends, impacts and drivers. Trends in ecology \& evolution, 25:345-53.

Potts, S. G. et al. (2016). Safeguarding pollinators and their values to human well-being. Nature, 540:220-229.

Sak, H., Senior, A. W., and Beaufays, F. (2014). Long short-term memory recurrent neural network architectures for large scale acoustic modeling. In INTERSPEECH, pages 338-342. ISCA.

Winston, M. (1991). The Biology of the Honey Bee. Harvard University Press. 